\title{
Rhythms and Robot Relations
}

\author{
Myriam Diocaretz $\cdot$ H. Jaap van den Herik
}

Published online: 6 August 2009

(C) The Author(s) 2009. This article is published with open access at Springerlink.com

\section{The First Rhythms}

Human-Robot Interaction has established itself as a research and development field within Robotics which has been in the mainstream for some decades. Since 2006, many conferences on Human-Robot Interaction have been held. ${ }^{1}$ They were the first rhythms. However, what so far did not enter the agenda and curricula is the personal and intimate relational dimension between a human and a robot.

This special issue shows the latest results on the research mentioned above. It contains seven carefully selected papers from the 1st International Conference on HumanRobot Personal Relationships ${ }^{2}$ (HRPR).

Robots and robotics in itself are not new. Robots are widely used in industrial machinery and the automotive industry, aerospace, the environment, transportation and logistics, and defence (especially the NASA). However, the focus of the new research is not on these industrial applications but rather on humanoid robots. These robots may act as mentor of ceremonies, guide, care taker, conductor, etc. The new re-

\footnotetext{
${ }^{1}$ For example, 1st Annual Conference on Human-Robot Interaction, March 2-4, 2006, Salt Lake City, Utah, USA (http://hri2006.org/). The theme of HRI 06, Toward Human-Robot Collaboration, highlighted the importance of creating robot capabilities and interfaces that addressed human concerns such as social appropriateness, safety, and quality of service.

${ }^{2}$ The conference was held on June 12-13, 2008 at Maastricht University in Maastricht, the Netherlands.
}

M. Diocaretz $(\bowtie) \cdot$ H.J. van den Herik

TiCC, Tilburg Centre for Creative Computing, Tilburg University, Warandelaan 2, 5037AB Tilburg, The Netherlands

e-mail: m.diocaretz@uvt.nl

H.J. van den Herik

e-mail: H.J.vdnHerik@uvt.nl search is also on robots (1) that can touch our heart, (2) that are designed to act as a potential companion, or (3) as an assistant, that may make one's daily life easier, and also, may perhaps give pleasure.

Within the fields of Human-Computer Interaction and Human-Robot Interaction, the past few years have witnessed a strong upsurge of interest in the more personal aspects of human relationships with artificial partners. Nowhere has this branch of interest been more strongly present and apparent than at Maastricht University, where the defence of a recent Ph.D. thesis by David Levy (2007), Intimate Relationships with Artificial Partners, and its subsequent book publication Love and Sex with Robots: The Evolution of Human-Robot Relationships attracted worldwide media publicity on an unprecedented scale.

Nowadays we find a number of state-of-the-art robots that are developed for some of the above-mentioned tasks. Of these robots, some are more sophisticated than others. Whatever the case, the increasing intervention and interaction between robots and humans in their (our) daily life environment make the Human-Robot relationship an urgent matter in need of critical and scientific attention.

\section{Robots and Rhythms}

Below we give six examples of robots and their possible rhythms in the actual world. As a first example we mention that there are one-dimensional robots, such as the Trio robot, which acted as master of ceremonies at the real South Korean wedding of Seok Gyeong-Jae, one of the engineers who designed it. Not only did the robot do his job well, but also there were additional small robots acting as guides 
for the guests. ${ }^{3}$ A second example comes from the Chinese Academy of Sciences in Beijing (August 4, 2006). They invented a female robot named RONG CHENG, programmed to speak the Sichuan dialect as she will be based at the Sichuan Science Museum to act as a receptionist and tour guide. $^{4}$ A third example is AsImo (Acronym for: Advanced Step in Innovative Mobility), made by Honda. It has been in development for 20 years, with the aim that it will be active at a home or in a hospital, or maybe as a firefighter. ${ }^{5}$ In 2004, AsIMO was learning how to run, then he learned how to find his way around obstacles as well as how to interact with people. In May 2008, he conducted the Detroit Symphony orchestra performing "The Impossible Dream" with some success. We considered this as a fourth example.

The fifth example is in medicine. It is important to recognise that there is a great potential for robots in e-Health and patient care. As a case in point we mention the $E d$ ucational and Therapeutic Toys for Autistic Children who are demonstrating to yield positive results in the AuRoRA project that uses therapy robots for individual treatments to motivate autistic children "to learn essential social skills such as turn-taking, joint attention and imitation." The sixth example is by the RовотA dolls. They are mini humanoid robots, who have been designed for "complex interaction with humans, involving speech, vision and body imitation" (cf. http://robota.epfl.ch/).

\section{Towards New Rhythms}

An important issue of the new developments is the social and humanistic side. It relates directly to the ethical and technological issues that are closely connected to Assistive Robotics, particularly in the world of individualised medicine. The robot-patient relationship remains the most popular and promising area for robots to be successfully accepted. However, the greatest challenge remains the area of human-robot personal relationships, which requires (1) face-to-face interaction, (2) direct connection, and (3) inherent trust in order to build love and friendship, including emotional attachment.

The human-robot experience must by necessity be immersive and fully functional to create a perception and experience of presence, activating psychophysical and neurological mechanisms in the human body. For interaction to happen in the human-robot personal relationship there is a need for visual, tactile, haptic, and auditory modalities; however,

\footnotetext{
${ }^{3}$ The manufacturer is Hanool Robotics. http://www.robotster.org/ page/2/

${ }^{4}$ http://rossignol.cream.org/?p=442

${ }^{5}$ http://newsvote.bbc.co.uk/mpapps/pagetools/print/news.bbc.co.uk/2/ hi/technology/6250621.stm
}

the most important factors reveal that for the new rhythms the ethical, social, and psychological aspects are paramount.

\section{The Articles}

On the whole, the papers in this special issue will discuss a number of major topics:

- Human ethics and roboethics $(2 \times)$,

- Evolutionary ethics and moral theory $(2 \times)$,

- Affective states: emotions and sentiments,

- Autonomous systems, robot design and affective mining, and

- Robotics and gender.

In The ethical treatment of artificially conscious robots, David Levy addresses the gaps in current research relating ethics and robotics. The focus within roboethics is specifically applied to robots possessing an artificial form of consciousness and the ethical treatment which these entities deserve. David Levy's arguments converge on the original proposal that conscious robots should have legal rights, a position which, no doubt, invites further debate.

Mark Coeckelberg presents an alternative approach to the ethics of personal robots in his contribution Personal robots, appearance, and the good: a methodological reflection on roboethics. He makes a case for the need to evaluate the potential impact of living with "personal" robots upon the humans' quality of life, from an anthropocentric perspective. He addresses roboethics as a form of applied ethics of relevance to designers. The article offers many questions (a) to delineate the alternative approach, which is defined as self-consciously anthropocentric instead of robocentric, and (b) to investigate more deeply the perspective of what the appearance of robots does to humans.

Transdisciplinary views between ethics, moral philosophy, and robotics design are demonstrated by two articles. In Evolutionary ethics in agent societies, Berend Berendsen and Pieter Spronck deal with ethical issues by exploring the research question whether multi-agent societies are able to evolve morally. They question whether a theory of evolutionary ethics provides a valid explanation for the existence of ethical behaviour. Thus, in their article, morality and moral sense are developed from a computational perspective. Subsequently, they provide a model of multi-agent societies. This model is applied to four experiments in which they investigate the agents' mating behaviour. They test the resulting rules and the circumstances within specific settings of family-relations and reputation. Their conclusions are encouraging and invite to further research.

In Combining moral theory, modal logic and MAS to create well-behaving artificial agents, Vincent Wiegel and Jan van den Berg link multi-agent systems (MAS) to moral philosophy and modal logic. They do so from a design focus. 
The authors address the need to give moral reasoning capabilities to autonomous software agents. For this purpose they explore (1) the notion of restraint and (2) the modes of detection of deviant behaviour. They arrive at the conclusion that analysing human moral reasoning to a considerable depth may lead to a useful model for constraining software agent behaviour.

In "I love this dog"-Children's emotional attachment to the robotic dog Aibo, Astrid Weiss, Daniela Wurhofer, and Manfred Tscheligi describe a study that they conducted to explore the first-time contact and the emotional attachment with a robotic pet. The article reports on a free exploration of a particular scene in a shopping mall assessing the emotional attachment of children and adults to the robotic pet AIBO. The AIBO robot dog was placed in the shopping area visited by many children and adults, whose immediate and automatic reactions to the dog were studied.

In Looking forward to sociable robots, Glenda ShawGarlock takes the subject of cultural factors as preconditions of robot assumptions that affect the design and, most importantly, the social use and the social practices. Her work provides a comparative and contrastive analysis of humanoid social robots in Japan and in North America. In particular, the vision behind two sociable robots projects is highlighted: KISMET (by Cynthia Breazeal at MIT) and REPLIÉE-Q2 (developed by Hiroshi Ishiguro at Osaka University). The contrast provides a framework for a cross-cultural analysis. The author investigates the underlying concepts and key factors, such as aesthetic appearance, social intelligence, morphology, and moral equivalence in each, and relates them to cultural and societal preconditions determining robot features. She concludes by suggesting that attention needs to be paid to the relevant societal pre-conditions when dealing with design choices. At that point she calls to mind Donna Haraway's feminist theory and technoscience perspective to stress the need to take responsibility in living and interacting with technology.

In Gender representation and humanoid robots designed for domestic use, Julie Carpenter, Joan M. Davis, Norah Erwin-Stewart, Tiffany R. Lee, John D. Bransford, and Nancy Vye concentrate on the promising and still little attended subject of robot-gender issues as an emerging field for future research in robotics design. Their focus is on the appearance and behaviour of androids, humanoid robots, and the role which gender representation may play in the expectations involved when human users integrate them in their daily home scenarios. They present the results of a study that reveals that androids designed for home use are likely to be assigned human roles and tasks. Thus, although the gender factor was not the central aim of their study but rather human roles, the conclusions drawn from the openended interviews demonstrate that human users are inclined to include gender issues in their expectations for the androids' different types of appearance and behaviour.

\section{Conclusions and Outlook for the Future}

The thought-provoking ideas on the relations between humans and robots of the future as published in the seven articles are by no means the end of the development. Yet, it is wise to formulate first our conclusions on what is achieved by the contents of this special issue and only then let our mind generate new ideas for future conferences and challenging research projects. From the above contents, it is clear that we may conclude that the time is ripe to reflect on the first steps in Human-Robot Personal Relationships research in terms of the effects upon humans. As a second conclusion we may state that we need guidelines on the issues involved in HRPR research. From the findings in the articles we may conclude that ethics is the major issue. It may range from personal human responsibility via the responsibility of designers and scientists to the responsibility to be assigned to robots.

Then, we may remark that the relations between humans and robots is an issue of ethics, too, and also of responsibilities. For instance:

(i) Is a human-robot relation acceptable in the real world?

(ii) Is it ethical to promote a delusion?

(iii) For the illusion of companionship and bonding, is such an illusion a short-term or a long-term one, and is it fair to create short-term illusions?

(iv) Do we want robots to evolve ethically?

From these three conclusions and the four questions posed above, the new research line is easy to foresee. We may predict the following four prevailing research questions for the next two to three years.

(a) What kind of robots and which social objectives do we want to support?

(b) Can a robot's existence be considered as equal to a person's and therefore be entitled to the same rights humans have?

(c) Is it important to define what the expectations concerning robots are?

(d) What are the different positions on using mind-andconsciousness approaches and on exploiting behavioural perspectives?

In summary, technology is changing our world and new rhythms will dominate our songs in such a way that swinging in the life of fashion will no longer discriminate between humans and robots.

Open Access This article is distributed under the terms of the Creative Commons Attribution Noncommercial License which permits any noncommercial use, distribution, and reproduction in any medium, provided the original author(s) and source are credited.

Myriam Diocaretz is the Socrates Professor, Special Chair in $\mathrm{Hu}-$ manism and Digital Society at Tilburg centre for Creative Computing 
(TiCC), Tilburg University. She is also the Secretary-General of the European Writers' Council (Brussels), the federation of authors' associations in 32 European countries. She received her M.A. in English from Stanford University, and Ph.D. in Comparative Studies from the State University of New York at Stony Brook (1982). She was a member of the Copyright Subgroup of the High Level Expert Group of the i2010 Digital Library initiative of the European Commission (INFSO \& Media) in 2006-2008. Since 2004 she has been selected as Independent Expert for the European Commission DG INFSO \& Media for project reviews and evaluations on digital culture and cultural heritage, access to and preservation of cultural and scientific resources, for the eTEN and eContentPlus programmes, as well as for the Social Sciences and Humanities Seventh Framework Programme (D-G Research). Her current lectures relate humanist visions and philosophy of technology with Knowledge Engineering.

Recent publications include: The Matrix in Theory (Editions Rodopi, 2006) with S. Herbrechter, and the essay "Interactivity and the New Technological Imaginary" (Acta Poetica 27, 2006). She has published research and led international projects on e-publishing, ICT innovation, e-content, Internet studies, eCulture, mobile services, and LivingLabs. She is the author, editor, and co-editor of 18 books in English, French, and Spanish on dialogical criticism, poetics, cultural and translation studies. She has founded five scholarly book series, including Critical Studies (Rodopi, Amsterdam/New York), which she directs since 1989.

H. Jaap van den Herik is Professor of Computer Science at Tilburg University, Tilburg, the Netherlands and Professor of Law and Computer Science at the Faculty of Law of the Leiden University, Leiden, the Netherlands. As of September 1, 2008 he is Director of TiCC. He was supervisor of 49 researchers, who successfully completed their theses. Together with Professor Maaike Meijer he was supervisor of David Levy's dissertation.

His research interests are: computer games, serious games, adaptive agents, neural networks, information retrieval, and intelligent systems for law applications.

At the moment, he is

- Editor-in-Chief of the ICGA Journal;

- Associate Editor of Transactions on Computational Intelligence and AI in Games;

- Associate Editor of the Entertainment Computing Journal;

- Member of the Royal Holland Society of Sciences and Humanities (since 1999); and

- Fellow of the European Coordinating Committee for AI (ECCAI). 